\title{
0 impacto da violência criminal urbana no preço dos imóveis residenciais na região da Tijuca, cidade do Rio de Janeiro: um estudo exploratório
}

Fernando José de Lacerda Carvalho Junior*

Celso Funcia Lemme**

\begin{abstract}
Resumo
Acompanhando a degradação ambiental e social dos grandes centros urbanos brasileiros, a questão da violência tem preocupado tanto os administradores públicos e privados, como os cidadãos em geral. Em 1998, o Banco Interamericano de Desenvolvimento calculou que os custos atribuídos à violência representavam, aproximadamente, $10,5 \%$ do PIB brasileiro. 0 presente estudo, de natureza exploratória, teve como objetivo estimar o impacto da violência criminal urbana sobre o preço dos imóveis residenciais na região da Tijuca, cidade do Rio de Janeiro, usando a metodologia de preços hedônicos. Foram utilizados dois modelos complementares, com amostras específicas distintas, para medir a segmentação do mercado imobiliário, o impacto gerado pelos crimes em diferentes regiões da cidade e a perda de valor dos imóveis residenciais pela proximidade de áreas de concentração do crime organizado. Os resultados encontrados, que devem ser considerados com cautela em função da natureza do estudo, confirmaram o que o senso comum indica e foram convergentes com os obtidos por outros estudos retratados na literatura internacional.
\end{abstract}

Palavras-chave: CADMA, meio ambiente, avaliação econômica, mercado imobiliário, violência urban

\begin{abstract}
Urban violence has become a major concern for politicians, corporate managers and ordinary people, following social and environmental degeneration in the largest cities in Brazil. In 1998, Inter-American Development Bank estimated that the total costs of urban violence for the Brazilian society were about $10,5 \%$ of the Gross Domestic Product. The objective of this paper was to estimate, with the support of a hedonic prices model, the impact of criminal urban violence on the market price of residential real estate in the district of Tijuca, city of Rio de Janeiro, Brazil. Two hedonic prices models have been used to evaluate both the impact of criminal violence in different districts of the city and the loss of value of houses and apartments due to the nearness of the slums in Tijuca. Main results were in accordance with common sense and previous studies. Readers must be advised that the results are very preliminary, because of the well-known problems with both the data on the Brazilian housing market and the hedonic prices model itself.
\end{abstract}

KEY WORDS : CADM A, Environment, Valuation, Real estate market, urban violence

\section{Introdução}

Nas ultimas décadas, a violência urbana nas grandes cidades brasileiras vem ganhando destaque cada vez maior no noticiário. As atenções da mídia estão voltadas, particularmente, para os conflitos entre traficantes, nas disputas por pontos de venda de drogas, e entre estes e a polícia, sem que a população consiga identificar claramente quem são os vencidos e os vencedores nessa luta. A favelização, a desagregação social e o desemprego contribuem para agravar esse quadro.

\footnotetext{
* Coordenador Financeiro, da Wilson, Sons. Mestre em Administração pelo Instituto COPPEAD de Administração, da Universidade Federal do Rio de Janeiro. E-mail: fernando.carvalho@ wilsonsons.com.br. Endereço: Rua Jardim Botânico, 518 - 20 andar - Jardim Botânico - RJ.

* Professor Adjunto do Instituto COPPEAD de Administração, da Universidade Federal do Rio de Janeiro. Doutor em Administração pelo Instituto COPPEAD de Administração, da Universidade Federal do Rio de Janeiro. E-mail: celso@ coppead.ufrj.br. Endereço: Prédio do COPPEAD. Rua 36, no 355 - Cidade Universitária - Ilha do Fundão. CEP: 21949-900 - Rio de Janeiro - RJ 
Em março de 1998, num congresso em Cartagena, na Colômbia, o Banco Interamericano de Desenvolvimento (BID) apresentou um estudo indicando que a violência era a principal barreira ao desenvolvimento da América Latina. Segundo o estudo do BID, o problema custava para os países da região o equivalente a $14,2 \%$ do Produto Interno Bruto (PIB), cerca de US\$168 bilhões, em valores da época. Para o Brasil, esse custo ficava em 10,5\% do PIB, equivalendo a US\$84 bilhões anuais.

A cidade do Rio de Janeiro tem estado no centro do noticiário policial, pela frequiência e gravidade dos atos criminosos. Sua topografia aproxima as comunidades carentes das classes mais favorecidas, uma vez que a cidade é praticamente rodeada de morros ocupados por favelas, com a maioria delas sendo controlada pelo crime organizado. $\mathrm{O}$ fechamento de importantes vias de tráfego por grupos criminosos, a utilização de armamento de grosso calibre de uso exclusivo das forças armadas por traficantes, o controle dos presídios por facções criminosas e a imposição de luto oficial por bandidos quando da morte de algum dos seus líderes tornaram-se rotina. Da mesma forma, virou lugar comum os desentendimentos entre as autoridades responsáveis pela área de segurança, nos diversos níveis de governo.

Este estudo, de natureza exploratória, procurou avaliar o impacto da violência criminal urbana no preço dos imóveis residenciais no município do Rio de Janeiro, mais especificamente na região da Tijuca, a partir de dados do mercado imobiliário do período de junho de 2000 a janeiro de 2002. A figura 1 destaca, no mapa do município do Rio de Janeiro, a área analisada.

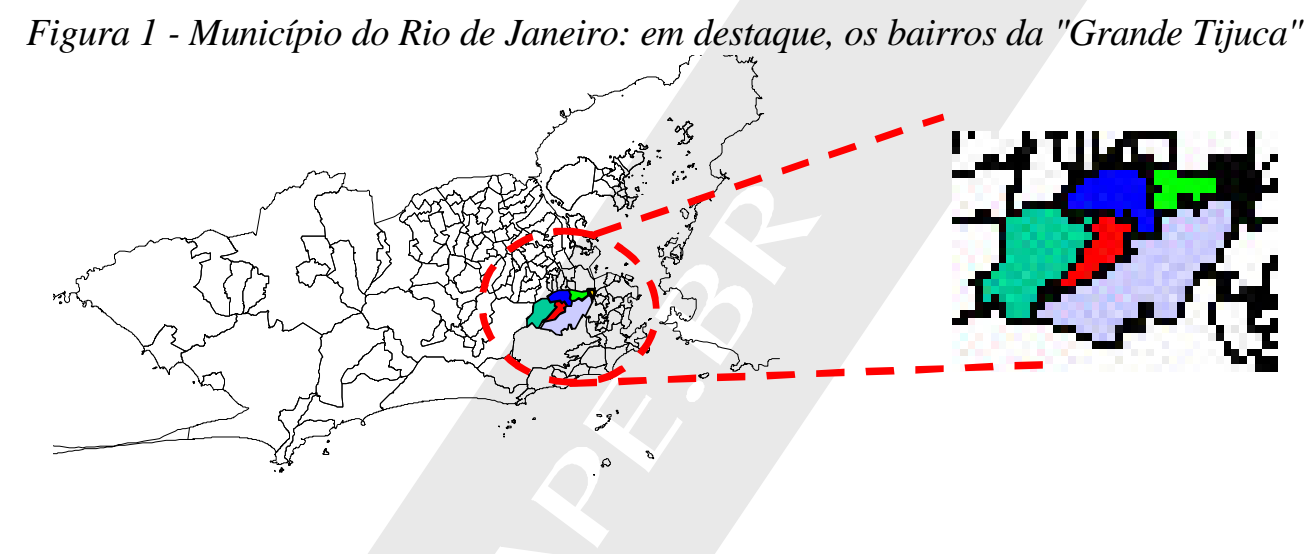

Fonte: CD-ROM Rio Atlas 97

Segundo Brueggeman e Fisher (1997, p.325), freqüentemente, o valor de uma propriedade é afetado pelas mudanças no ambiente ou pelo conhecimento de que esse ambiente tenha se tornado potencialmente danoso. Aqui, foi utilizado o método de preços hedônicos (do inglês hedonic prices) para determinar a perda de valor dos imóveis em função da localização e da qualidade da circunvizinhança. Foram excluídos do trabalho imóveis comerciais, de qualquer espécie, como escritórios, lojas e armazéns.

O município do Rio de Janeiro, com uma população de 5.857.904 habitantes - pelo censo de 2000 do Instituto Brasileiro de Geografia e Estatística (IBGE) -, tem densidade demográfica de 4.667 habitantes $/ \mathrm{km}^{2} \mathrm{e}$ apresentou nesse mesmo, segundo dados da prefeitura, uma taxa de 848 "principais delitos" por 100.000 habitantes, desigualmente divididos pelas Áreas de Planejamento(AP) da cidade. São definidos como "principais delitos", o homicídio doloso, o latrocínio, o seqüestro, o estupro, o furto e o roubo de veículos, bem como o roubo a transeunte, residências, instituições financeiras e em transportes coletivos. Entende-se por AP as cinco subdivisões da cidade, que consolidam as regiões administrativas.

O bairro da Tijuca, foco deste estudo, faz parte da AP 2 e tem alto índice de criminalidade, somando-se os crimes contra a vida (CCV) e os crimes contra o patrimônio(CCP). Os morros do bairro abrigam diversas favelas controladas pelo crime organizado, como as do Borel, Formiga, Turano e Salgueiro. A figura 2 destaca os mapas dos bairros que compõem a "Grande Tijuca" e as principais favelas. 


\section{Figura 2 - "Grande Tijuca" (esquerda) e principais favelas (direita)}

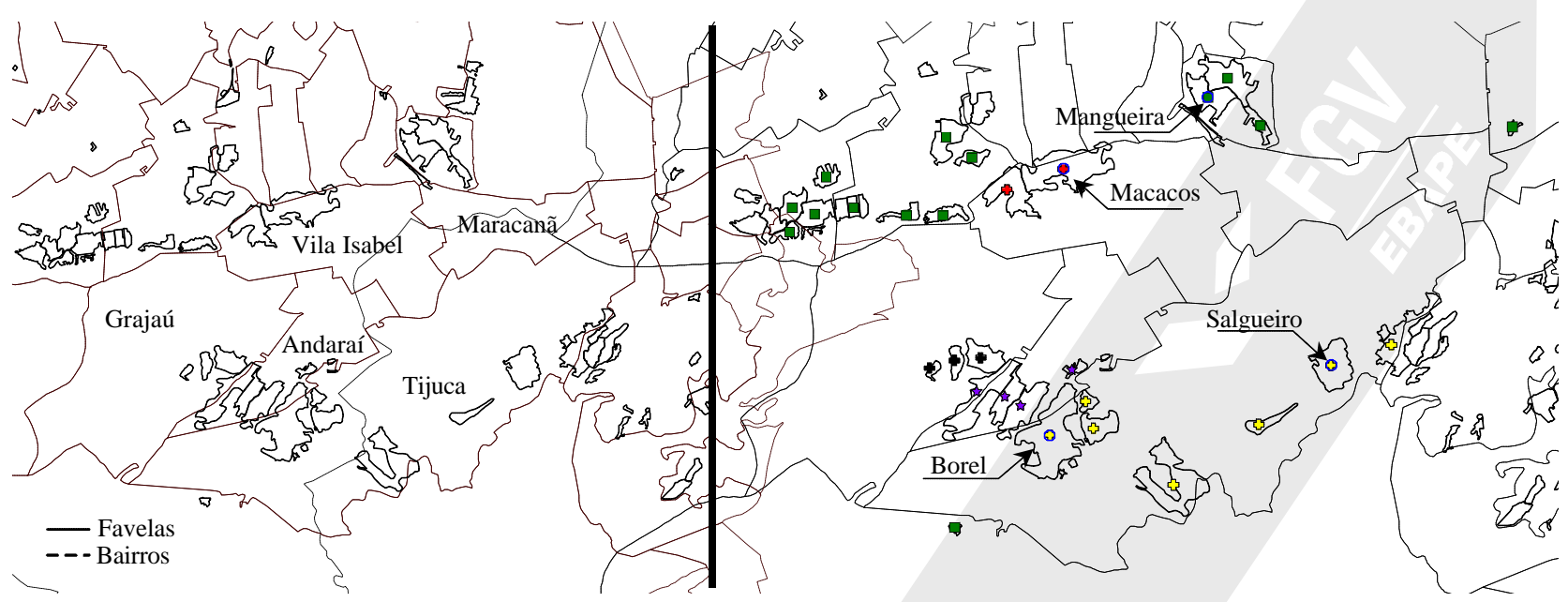

Fonte: CD-ROM Rio Atlas 97

A Secretaria de Segurança divide o estado do Rio de Janeiro em Áreas Integradas de Segurança Pública (AISP), que representam grupos de delegacias de polícia administrados pelo governo estadual; a Tijuca está incluída na AISP 6. Inicialmente, o foco do estudo era exclusivamente o bairro da Tijuca, definido pelo limite oficial de bairros do município do Rio de Janeiro. No entanto, por questões de acesso aos dados, foram incluídos imóveis dos bairros vizinhos, como Andaraí, Grajaú, Maracanã e Vila Isabel, utilizando-se o conceito de "Grande Tijuca", daqui em diante referido simplesmente como Tijuca.

Pelo caráter experimental do estudo e devido à segmentação do mercado imobiliário, foram traçados dois modelos de análise para caracterizar o impacto da criminalidade na Tijuca. O modelo 1 , com dados mais agregados, considera informação sobre imóveis de diferentes bairros do município, na tentativa de se calcular um diferencial de violência da Tijuca em relação ao restante da cidade, batizado de "delta Tijuca". O modelo 2 , com dados mais detalhados de imóveis da área da Tijuca, foi utilizado para estimar o custo da violência resultante da proximidade de áreas com concentração de criminalidade (favelas). Em seguida, o artigo revê alguns conceitos básicos para o desenvolvimento desta pesquisa. Mais adiante, é detalhada a metodologia empregada; na seção seguinte são apresentados os resultados e, na conclusão, são feitas sugestões para futuras pesquisas.

\section{Revisão da literatura}

$\mathrm{Na}$ avaliação de propriedades, o objetivo é estabelecer um valor de mercado; geralmente, o preço que mais provavelmente seria pago em mercados competitivos. A noção de valor é diferente da de preço, uma vez que, devido às preferências pessoais, os preços individuais podem se afastar daquele que seria pago pelo mercado (BRUEGGEMAN e FISHER, 1997, p.184-193). Além disso, o avaliador deve examinar o submercado geralmente, incluindo a vizinhança, o comércio da região, as escolas e outros aspectos - para estabelecer algum prêmio ou desconto a ser associado à propriedade, em função da qualidade da comunidade que a cerca. Desse ponto em diante, como é comum entre os profissionais do mercado imobiliário, utilizaremos indistintamente os termos "preço" e "valor", apesar da diferença conceitual existente entre eles.

Brueggeman e Fisher (1997) entendem que, para estimar o valor de uma propriedade, o avaliador pode levar em conta os preços de mercado, o custo de reposição e a receita gerada. No caso de imóveis residenciais, sugerem que considerar os preços de mercado e o custo de reposição possibilita estimativas mais confiáveis. No primeiro caso, também chamado de "método de comparação" de vendas (sales comparison approach), é 
possível identificar propriedades que tenham sido vendidas mais recentemente e sejam comparáveis à propriedade em questão, sendo feitos ajustes devido às diferenças entre elas, que devem ser as mínimas possíveis para manter a comparabilidade. No segundo caso, considerando-se o custo de reposição, pelo chamado "método de custos" (cost approach), a intenção é estimar o custo de reproduzir a estrutura física (menos a depreciação) e somá-lo o valor do terreno para se obter o valor total.

O método de preços hedônicos busca estimar o valor de um bem ou serviço que não seja negociado em mercado, pela observação dos preços de bens ou serviços a ele associados (FREEMAN, 1993). Preços de imóveis, terras e salários, por exemplo, são utilizados para atribuir valores implícitos a fatores ambientais, estruturais e qualitativos, entre outros difíceis de valorar. Dixon, Scura e Carpenter (1994) destacam que a noção de preços implícitos é antiga, observando que os economistas, muito antes da aplicação da teoria dos preços hedônicos, já documentaram a relação entre os preços de imóveis e algumas características ambientais. Segundo Rosen (1974), uma classe de produtos diferenciados pode ser completamente descrita por um vetor de características objetivamente medidas. Dessa maneira, a identificação de preços de produtos e de características específicas a eles associadas pode ser usada para definir um grupo de preços hedônicos (implícitos).

Dois artigos estudados abordam especificamente critérios de vizinhança dos imóveis residenciais e foram utilizados nesta pesquisa como base metodológica. Cummings, Dipasquale e Kahn (2001) estudaram os complexos Nehemiah, empreendimentos habitacionais para a classe média, localizados em vizinhanças afligidas (áreas que já sofriam com degradação urbanística e altos índices de criminalidadena cidade de Filadélfia, EUA, construídos com subsídios governamentais para promover o desenvolvimento da região. Ao escolher os complexos Nehemiah, os residentes mudaram para comunidades consideravelmente piores do que aquelas em que moravam quando eram inquilinos. Uma das variáveis específicas que os autores consideraram para explicar o preço dos imóveis foi a exposição ao crime, delimitada pelo setor censitário ao qual os complexos pertenciam. Nos complexos Nehemiah, os novos proprietários conseguiram maior qualidade nas variáveis estruturais de suas residências, mas com maior exposição ao crime e dispondo de fraco sistema público de ensino.

Clark e Cosgrove (1990) usaram modelos de preços hedônicos interurbanos para estimar especificamente a demanda por segurança pública. Eles utilizaram como medida de segurança pública o inverso da taxa de exposição a crimes, mais especificamente homicídios. Esses autores indicam o trabalho de Thaler (1978 apud CLARK e COSGROVE, 1990) como seminal na utilização de modelos hedônicos para medir segurança pública.

Por ser um método fortemente quantitativo e baseado em ferramental estatístico, a abordagem de preços hedônicos exige grande quantidade de dados sobre preços de venda de unidades individuais, bem como a coleção de suas características físicas (locacionais, ambientais etc.), o que tem limitado seu uso (FREEMAN, 1993). Procura-se, na medida do possível, utilizar dados de vendas reais para os preços dos imóveis, ainda que muitos autores baseiem suas análises em dados de censo, mais fáceis de serem obtidos. Uma alternativa para a obtenção de dados seria a avaliação por profissionais (DIXON, SCURA e CARPENTER, 1994).

Além do volume de informações necessárias, para que os modelos possam ser significativos, Butler (1982 apud MACEDO, 1998) ressalta que dados de muitas das características dos imóveis não estão disponíveis ou são de baixíssima qualidade. Destaca, ainda que, além da restrição dos dados, a formação intrínseca de grupos (clusters) em combinações de características leva a uma considerável multicolinearidade nas estimativas que empregam uma seleção generosa de variáveis explicativas; questão também levantada por Dixon, Scura e Carpenter (1994).

Bowen, Mikelbank e Prestegaard (2001), ao discutirem o conjunto de variáveis para os modelos de preços hedônicos, apontam a necessidade de que todo o conjunto de atributos determinantes dos preços seja incluído, tanto os referentes ao custo quanto os que representam utilidade aos compradores. Porém, esse conjunto não pode ser definido a priori apenas com base na teoria, pois envolve julgamento e experiência com o mercado de 
imóveis. Esses autores indicam três classes de atributos, que seriam determinantes do preço: as estruturais, as ambientais e as locacionais. Por sua vez, Ding, Simons e Baku (2000) ampliam e detalham essas classes:

- $\quad$ estruturais - tipo de imóvel, número de quartos, área útil e vagas na garagem;

- locacionais - distância do centro de negócios, proximidade de lojas e shoppings e qualidade do sistema de transporte;

- de vizinhança - presença de boas escolas, segurança pública e serviços públicos;

- ambientais - níveis de poluição do ar e sonora, boa ou má vista etc.

Para identificar os atributos usados mais freqüentemente, Wilhelmsson (2000) investigou todos os artigos relacionados a preços hedônicos publicados no Journal of Real Estate Research e no Journal of Urban Economics entre 1990 e 1995 (28 no total), encontrando os resultados indicados na tabela 1.

Tabela 1 - Atributos por ordem decrescente de utilização das variáveis nos artigos empíricos do Journal of Real Estate Research

(1990-95)

\begin{tabular}{lcc}
\hline \multicolumn{1}{c}{ VARIÁVEL } & JOURNAL OF REAL ESTATE RESEARCH & JOURNAL OF URBAN ECONOMICS \\
\hline Área construída & $81 \%$ & $83 \%$ \\
\hline Número de banheiros & $75 \%$ & $58 \%$ \\
\hline Vagas de garagem & $63 \%$ & $67 \%$ \\
\hline Área do terreno & $56 \%$ & $75 \%$ \\
\hline Número de quartos & $50 \%$ & $42 \%$ \\
\hline Idade do imóvel & $50 \%$ & $75 \%$ \\
\hline Lareira & $44 \%$ & $50 \%$ \\
\hline Ar condicionado & $25 \%$ & $50 \%$ \\
\hline Julgamento subjetivo & $25 \%$ & $33 \%$ \\
\hline Piscina & $19 \%$ & $17 \%$
\end{tabular}

Fonte: Wilhelmsson (2000)

Muitos estudos apontam a distância (em relação à fonte das externalidades) como uma variável importante na análise. Num estudo sobre novos investimentos e seu impacto no valor dos imóveis, Ding, Simons e Baku (2000) postularam como sua primeira hipótese a redução do efeito com a distância, o que foi confirmado pelos resultados. Isso contradiz os resultados do estudo realizado por Simons (1998 apud DING, SIMONS e BAKU, 2000), podendo a divergência ser devida à avaliação pouco precisa das distâncias.

Outro aspecto sujeito a controvérsias é a forma funcional da equação de preços hedônicos. Como explicita Rosen (1974 apud WILHELMSSON, 2000), isso tem sido objeto de debate entre os pesquisadores, mas a teoria econômica falha em indicar alguma forma particular como mais apropriada, uma deficiência parece ser percebida por diversos pesquisadores (DIXON, SCURA e CARPENTER, 1994; MACEDO, 1998; TU e EPPLI, 1999; BOWEN, MIKELBANK e PRESTEGAARD, 2001). Para Macedo (1998), a maioria dos pesquisadores vê esse ponto como uma questão empírica, a ser decidida pelo critério da qualidade de ajustamento(goodness-of-fit). Tu e Eppli (1999) entendem que a teoria econômica coloca poucas restrições quanto à forma funcional e que, pela evidência empírica, nenhuma forma funcional domina completamente as outras. 


\section{Metodologia}

Como anteriormente mencionado, neste estudo foram utilizados dois modelos com abordagens distintas e complementares. O primeiro (modelo 1) buscou medir o impacto da violência criminal no preço dos imóveis em diferentes bairros da cidade, para poder ter uma estimativa do diferencial resultante da incidência de crimes na Tijuca, batizado de "delta Tijuca". O segundo (modelo 2) procurou indicar, para imóveis da Tijuca, impactos específicos (além do delta Tijuca) pela proximidade das áreas subnormais (favelas).

\section{Modelos de preços hedônicos}

O modelo 1 utilizou a segmentação da cidade por bairros, para verificar a existência de impactos diferenciados de violência criminal (deltas) estatisticamente significativos entre eles. As variáveis instrumentais para medir a violência foram os dados agregados de números de crimes por Áreas Integradas de Segurança Pública (AISP), com base na metodologia de Clark e Cosgrove (1990). Esse modelo é mais impreciso, incluindo dados de oferta de imóveis e menor número de variáveis explicativas, devido à quantidade e qualidade dos dados obtidos (acessibilidade). As variáveis independentes foram divididas em estruturais, de localização e de vizinhança. A tabela 2 relaciona e descreve as variáveis incluídas no modelo 1 :

Tabela 2 - Modelo 1

\begin{tabular}{|c|c|}
\hline VARIÁVEL DEPENDENTE & DESCRIÇÃ̃O \\
\hline Preço & Preço de oferta de venda \\
\hline VARIÁVEIS ESTRUTURAIS DOS IMÓVEIS & DESCRIÇÃO \\
\hline Quartos (+) & $\mathrm{N}^{\mathrm{o}}$ de quartos \\
\hline Andar (+) & $\mathrm{N}^{\mathrm{o}}$ de andares (linear, dúplex, tríplex) \\
\hline Cobertura (+) & Variável dummy, um para cobertura \\
\hline Suíte $(+)$ & № de suítes \\
\hline Lavabo (+) & $\mathrm{N}^{\mathrm{o}}$ de lavabos \\
\hline Playground (+) & Variável dummy, um para área de lazer \\
\hline Garage (+) & $\mathrm{N}^{\mathrm{o}}$ de vagas de garagem (na escritura). \\
\hline M2 (+) & Área $\left(\mathrm{em} \mathrm{m}^{2}\right)$ \\
\hline VARIÁVEIS DE LOCALIZAÇÃO & DESCRIÇÃO \\
\hline Bairros (?) & $\begin{array}{l}\text { Vetor de variáveis dummies, cada variável repre- } \\
\text { sentando um bairro, um para o bairro. }\end{array}$ \\
\hline VARIÁVEIS DE VIZINHANÇA & DESCRIÇÃ̃O (por AISP do bairro) \\
\hline $\operatorname{CCV}(-)$ & $\mathrm{N}^{\mathrm{o}}$ de crimes contra a vida. \\
\hline CCP (-) & $\mathrm{N}^{\mathrm{o}}$ de crimes contra o patrimônio \\
\hline CLD (-) & $\mathrm{N}^{\mathrm{o}}$ de crimes ligados às drogas \\
\hline EC (-) & № de encontro de cadáveres \\
\hline PDA (-) & № de ocorrências de porte e disparo de armas. \\
\hline
\end{tabular}

$(+)=$ o sinal esperado para os coeficientes de regressão.

Fonte das variáveis estruturais: $O$ Globo (cadernos "Classificados" e "Morar bem") e sites de imobiliárias.

O modelo 2 incluiu apenas imóveis da Tijuca, com maior número de variáveis independentes. Tenta estimar, através da distância euclidiana (linha reta, em referência à "linha de tiro") entre os imóveis e os centróides das favelas, o impacto da violência criminal no preço dos imóveis residenciais. Além disso, buscou incorporar dados de negociações efetivas, retirados dos laudos de imóveis vendidos. Quanto às variáveis, estas foram baseadas na literatura pesquisada, nas entrevistas com corretores experientes, nos laudos dos imóveis e no mapa do município do Rio de Janeiro. A tabela 3 relaciona e descreve essas variáveis. 
Tabela 3 - Modelo 2

\begin{tabular}{|c|c|c|}
\hline VARIÁ VEL DEPENDENTE & DESCRIÇÃO & FONTE \\
\hline Preço & Preço efetivo de venda & Laudos de imobiliárias \\
\hline VARIÁ VEIS ESTRUTURAIS & DESCRIÇÃO & FONTE \\
\hline Idade (-) & № de anos desde a construção & Laudos de imobiliárias \\
\hline Quartos (+) & $\mathrm{N}^{\mathrm{o}}$ de quartos & Laudos de imobiliárias \\
\hline Suítes (+) & № de suítes & Laudos de imobiliárias \\
\hline Banheiro social $(+)$ & № de banheiros sociais & Laudos de imobiliárias \\
\hline Lavabo (+) & № de lavabos & Laudos de imobiliárias \\
\hline Andar (+) & Andar do imóvel (casa = um) & Laudos de imobiliárias \\
\hline Posição (?) & $\begin{array}{l}\text { Variável dummy - frente = um, } \\
\text { fundos }=0\end{array}$ & Laudos de imobiliárias \\
\hline Área utilizada (+) & № de $\mathrm{m}^{2}$ de área útil & Laudos de imobiliárias \\
\hline Vagas $(+)$ & $\mathrm{N}^{\mathrm{o}}$ de vagas de garagem na escritura & Laudos de imobiliárias \\
\hline Sauna (+) & Variável dummy, sauna = um & Laudos de imobiliárias \\
\hline Playground (+) & Variável dummy, playground = um & Laudos de imobiliárias \\
\hline Piscina (+) & Variável dummy, piscina $=$ um & Laudos de imobiliárias \\
\hline VARIÁVEIS DE LOCALIZAÇÃO & DESCRIÇÃO & FONTE \\
\hline Facilidades $(+)$ & $\begin{array}{l}\text { Variável dummy, facilidades = uma } \\
\text { indica proximidade a transporte }\end{array}$ & Laudos de imobiliárias \\
\hline CEPS (?) & $\begin{array}{l}\text { Vetor de variáveis dummies, indicando } \\
\text { CEP do imóvel }\end{array}$ & $\begin{array}{l}\text { Site da Empresa de Correios } \\
\text { e Telégrafos (ECT) }\end{array}$ \\
\hline VARIÁVEIS DE VIZINHANÇA & $\begin{array}{l}\text { DESCRIÇÃO } \\
\text { Distância em Km }\end{array}$ & FONTE \\
\hline BOR_KM (+) & Do imóvel ao Morro do Borel & CD-ROM RioAtlas98 \\
\hline SAL_KM (+) & Do imóvel ao Morro do Salgueiro & CD-ROM RioAtlas98 \\
\hline MAC_KM (+) & Do imóvel ao Morro dos Macacos & CD-ROM RioAtlas98 \\
\hline MANG_KM & Do imóvel ao Morro da Mangueira & CD-ROM RioAtlas98 \\
\hline FAV5_KM (+) & $\begin{array}{l}\text { Do imóvel ao centróide da favela mais } \\
\text { próxima (fora as quatro anteriores) }\end{array}$ & CD-ROM RioAtlas98 \\
\hline DMIN_KM (+) & Menor valor das variáveis 1 a 5 & \\
\hline DMIN4_KM (+) & Menor valor entre as variáveis 1 a 4 & \\
\hline PRODTOT (+) & Produto das variáveis $1,2,3,4$ e 5 & \\
\hline PROD_4 (+) & Produto das variáveis $1,2,3$ e 4 & \\
\hline
\end{tabular}

$(+)=$ expectativa do sinal dos coeficientes de regressão.

Nos dois modelos, as variáveis estruturais foram escolhidas com base na literatura, principalmente, no já mencionado trabalho de Wilhelmsson (2000). Além disso, entrevistas com experientes corretores indicaram a inclusão de variáveis referentes ao andar do imóvel (caso seja apartamento) e à posição do apartamento em relação à rua (frente ou fundos).

A variação sistemática nos preços de venda dos imóveis depende da sua localização na região de estudo (BOWEN, MIKELBANK e PRESTEGAARD, 2001); assim, no modelo 1 parte-se da hipótese de que imóveis do mesmo bairro teriam as mesmas características de localização (inclusão do vetor de variáveis dummies dos bairros). Essa suposição decorre, basicamente, da falta de informações detalhadas sobre cada imóvel. Entretanto, como indica o senso comum, há diferenças intrabairros (como proximidade da praia, belas vistas, proximidade de favelas etc.) que não são contempladas nesse modelo. O modelo 2, com a inclusão do vetor de variáveis dummies Código de Endereçamento Postal (CEP), busca apreender pequenas diferenças na localização, levando em conta que num mesmo bairro coexistem áreas mais nobres com outras mais desvalorizadas. Ding, Simons e Baku (2000) e Tu e Eppli(1999) são exemplos de trabalhos que recorrem a essas variáveis para apreender tais nuances.

As variáveis instrumentais que procuram medir o custo da violência criminal urbana no modelo 1 são os dados agregados de crimes por Área Integrada de Segurança Pública. Para o modelo 2, as variáveis instrumentais são as distâncias entre os imóveis e as quatro áreas subnormais consideradas; ou seja, os morros do Borel, 
Salgueiro, Macacos e Mangueira, além da distância a uma quinta área subnormal, distinta das quatro anteriores, que fosse mais próxima de cada imóvel da amostra (BOR_KM, SAL_KM, MAC_KM, MANG_KM e FAV5_KM, respectivamente). Além disso, foram incluídas as variáveis DMIN_KM e DMIN4_KM, que representam a menor distância entre o imóvel e uma das cinco áreas subnormais e a menor distância entre o imóvel e uma das quatro principais áreas subnormais, respectivamente. Foram ainda incluídas duas variáveis (PRODTOT e PROD_4) que procuraram medir o impacto conjunto das cinco áreas subnormais e das quatro principais áreas subnormais, com seus respectivos valores obtidos pelo produto das distâncias entre os imóveis e as cinco áreas subnormais e entre eles e as quatro principais áreas subnormais, respectivamente.

Para o modelo 1, a expectativa era que os coeficientes das variáveis de vizinhança fossem negativos e significativos (quanto mais crimes, menor o valor dos imóveis) e para o modelo 2, que fossem positivos e significativos, indicando que quanto maior a distância, maior o valor do imóvel.

\section{Amostras dos imóveis}

Como já dito antes, foram utilizadas duas amostras na pesquisa, para os modelos 1 e 2 . O estudo não teve acesso a uma base pronta de dados, com informações organizadas sobre a negociação dos imóveis. Por isso, teve que montar as amostras a partir de diversas fontes de informação. Clark e Cosgrove (1990) também comentam a variedade de fontes de dados em seu estudo. A tabela 4 resume os principais tipos e fontes de informação:

Tabela 4 - Principais tipos e fontes de informação

\begin{tabular}{|c|c|}
\hline TIPOS DE INFORMAÇÃO & FONTES \\
\hline $\begin{array}{l}\text { 1. Relação de corretoras de imóveis atuantes no } \\
\text { bairro da Tijuca, para obtenção de dados de negoci- } \\
\text { ações de imóveis. }\end{array}$ & $\begin{array}{l}\text { Entidades de classe (Abadi, Secovi e Ademi), Fede- } \\
\text { ração das Indústrias (Firjan) e fontes de acesso pú- } \\
\text { blico (Internet e Listas Amarelas). }\end{array}$ \\
\hline $\begin{array}{l}\text { antes à formação de preços, para } \\
\text { áveis independentes do modelo de }\end{array}$ & $\begin{array}{l}\text { Corretores de imóveis experientes, através de entre- } \\
\text { vistas focalizadas. }\end{array}$ \\
\hline $\begin{array}{l}\text { 3. Preços de venda e características estruturais dos } \\
\text { imóveis. }\end{array}$ & $\begin{array}{l}\text { de } \\
\text { al do }\end{array}$ \\
\hline 4. Características locacionais e ambientais. & $\begin{array}{l}\text { Censo } 2000 \text { (IBGE), estatísticas criminais do muni- } \\
\text { cípio e do estado. Distância entre áreas subnormais } \\
\text { e os imóveis da amostra (Instituto Pereira Passos - } \\
\text { PCRJ); e estatísticas gerais da cidade, no site } \\
\text { <www.armazémdedados.rio.rj.gov.br). }\end{array}$ \\
\hline
\end{tabular}

O item 2 foi comum a ambas amostras. Foi coberto por duas entrevistas, sem um questionário formal, com dois experientes profissionais do ramo imobiliário. BRUEGGEMAN e FISHER (1997) julgam importante a opinião do profissional experiente, pois seu julgamento e o conhecimento das condições do mercado influenciam decisivamente os preços. O primeiro entrevistado (C1) foi um corretor autônomo, atuante numa das imobiliárias mais destacadas do mercado imobiliário residencial; o segundo(C2) foi o diretor regional da loja do bairro da Tijuca de outra grande imobiliária, também corretor experiente no ramo.

\section{Tratamento dos dados}

Os dados foram tratados conforme a metodologia de preços hedônicos usual na literatura, utilizando-se os softwares Microsoft ${ }^{\circledR}$, Excel 97 e SPSS for Windows 10.0.1. Para o modelo 1, foram calculadas as estatísticas descritivas e eliminados outliers, com base no Índice de Cook's (BENSON, McCLAVE e SINCICH, 2002) e em julgamento qualitativo, seguindo-se o exame de multicolinearidade pelo Índice de Tolerância (1-R2). As estatísticas descritivas - principalmente, do preço de oferta - mostraram uma amostra concentrada em imóveis de valor menor que a média, mas com grande dispersão, indicada pela elevada curtose e pelo alto desvio padrão. Foram realizadas 12 regressões, variando a forma funcional da equação de regressão, o método 
utilizado e as variáveis independentes, observando-se as estatísticas $\mathrm{R}^{2}$ ajustado e $F$, além das análises dos resíduos pelo teste de Kolmogorov-Smirnov. Ao final, foram selecionadas as equações de regressão que apresentavam as estatísticas mais consistentes.

Para o modelo 2, foram feitas as mesmas análises básicas do modelo 1 (outliers, multicolinearidade e normalidade de resíduos), seguidas de 18 análises de regressão com a função LINEAR - nove delas pelo método ENTER e nove pelo STEPWISE - nas quais foram incluídas, individualmente, cada uma das nove variáveis de vizinhança. Em seguida, foram selecionadas as equações de regressão cujas variáveis de vizinhança foram incluídas pelo método STEPWISE. Com elas foram realizadas regressões com a forma funcional SEMI-LOG, pelo método STEPWISE. Por fim, foi comparada a estatística R2ajustado das equações nas formas funcionais LINEAR e SEMI-LOG, optando-se por analisar os resultados das regressões na forma LINEAR.

Neste estudo, foi utilizada apenas a forma linear, de interpretação direta (o coeficiente indica diretamente o valor da disposição marginal a pagar pela característica em questão), e a forma SEMI-LOG, que mostra a variação percentual no preço em função da variação de uma unidade da característica. Não foram utilizadas as formas LOG-LOG e BOX-COX, devido à inclusão de variáveis dicotômicas, que podem assumir o valor zero, cujo logaritmo é indeterminado. Linneman (1980, apud TU e EPPLI, 1999) sugere que essas formas não são adequadas a variáveis independentes que sejam dicotômicas, como ocorre em ambos os modelos utilizados. 


\section{Resultados}

\section{Modelo 1}

A tabela 5 resume os resultados das regressões realizadas com esse modelo.

Tabela 5 - Resumos dos resultados do modelo 1

\begin{tabular}{|c|c|c|c|c|c|c|c|c|c|}
\hline & \multirow{5}{*}{$\begin{array}{l}\text { Função } \\
\text { Método } \\
\mathrm{R}^{2} \text { ajust } \\
\mathrm{F}\end{array}$} & \multicolumn{4}{|c|}{ LINEAR } & \multicolumn{4}{|c|}{ SEMI-LOG } \\
\hline & & \multicolumn{2}{|c|}{ ENTER } & \multicolumn{2}{|c|}{ STEPWISE } & \multicolumn{2}{|c|}{ ENTER } & \multicolumn{2}{|c|}{ STEPWISE } \\
\hline & & \multicolumn{2}{|c|}{$76,9 \%$} & \multicolumn{2}{|c|}{$76,8 \%$} & \multicolumn{2}{|c|}{$88,5 \%$} & \multicolumn{2}{|c|}{$88,4 \%$} \\
\hline & & \multicolumn{2}{|c|}{126,1} & \multicolumn{2}{|c|}{215,4} & \multicolumn{2}{|c|}{288,3} & \multicolumn{2}{|c|}{389,2} \\
\hline & & p-values & coefic. & p-values & coefic. & p-values & coefic. & p-values & coefic. \\
\hline & Construção & $10,9 \%$ & 104.616 & $0,3 \%$ & 147.059 & $0,0 \%$ & 5,20 & $0,0 \%$ & 5,89 \\
\hline \multirow{8}{*}{ 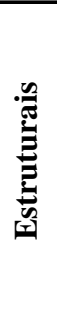 } & Área & $0,0 \%$ & 2.609 & $0,0 \%$ & 2.763 & $0,0 \%$ & 0,00 & $0,0 \%$ & 0,00 \\
\hline & Quartos & $0,0 \%$ & $(71.246)$ & $0,0 \%$ & $(70.204)$ & $0,0 \%$ & 0,09 & $0,0 \%$ & 0,09 \\
\hline & Suítes & $2,0 \%$ & 30.642 & $\begin{array}{ll}---- \\
\end{array}$ & ----- & $0,0 \%$ & 0,04 & $0,0 \%$ & 0,04 \\
\hline & Garagem & $0,0 \%$ & 104.548 & $0,0 \%$ & 102.117 & $0,0 \%$ & 0,07 & $0,0 \%$ & 0,07 \\
\hline & Andar & $16,6 \%$ & 49.533 & ----- & ----- & $87,8 \%$ & 0,00 & ----- & ----- \\
\hline & Cobertura & $0,0 \%$ & (190.334) & $0,0 \%$ & (175.770) & $13,0 \%$ & 0,04 & ----- & ----- \\
\hline & Lazer & $17,7 \%$ & $(24.319)$ & ----- & ----- & $0,0 \%$ & 0,05 & $0,1 \%$ & 0,04 \\
\hline & Lavabo & $0,0 \%$ & 128.816 & $0,0 \%$ & 132.358 & $0,1 \%$ & 0,05 & $0,0 \%$ & 0,05 \\
\hline \multirow{12}{*}{ 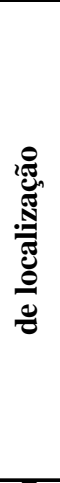 } & Laranjeiras & $42,5 \%$ & 26.078 & ----- & ----- & $0,0 \%$ & 0,09 & $0,5 \%$ & 0,06 \\
\hline & Botafogo & $41,2 \%$ & 26.885 & ----- & ----- & $4,6 \%$ & 0,04 & ----- & ----- \\
\hline & Copacabana & ----- & ----- & ----- & ----- & ----- & ----- & $0,0 \%$ & $(0,24)$ \\
\hline & Ipanema & $0,0 \%$ & 432.594 & $0,0 \%$ & 465.732 & $0,0 \%$ & 0,25 & ----- & +---- \\
\hline & Leblon & $0,0 \%$ & 323.661 & $0,0 \%$ & 317.686 & $0,0 \%$ & 0,26 & ----- & ----- \\
\hline & Lagoa & $0,4 \%$ & 107.696 & $0,2 \%$ & 103.664 & $0,0 \%$ & 0,15 & $0,0 \%$ & $(0,12)$ \\
\hline & Barra & $5,8 \%$ & $(69.580)$ & ----- & ----- & $0,5 \%$ & 0,07 & $0,2 \%$ & $(0,06)$ \\
\hline & Recreio & $0,0 \%$ & (176.542) & $0,0 \%$ & (115.287) & $29,3 \%$ & $(0,03)$ & $0,0 \%$ & $(0,17)$ \\
\hline & Jacaré & ----- & ---- & ---- & ----- & ----- & ----- & ----- & ----- \\
\hline & Tijuca & $5,3 \%$ & $(64.070)$ & ----- & ----- & $0,0 \%$ & $(0,08)$ & ----- & ----- \\
\hline & Maracanã & $59,6 \%$ & (17.409) & ----- & ----- & $0,0 \%$ & $(0,12)$ & ----- & ----- \\
\hline & Grajaú & $12,3 \%$ & $(51.158)$ & ----- & ----- & $0,0 \%$ & $(0,15)$ & $1,0 \%$ & $(0,06)$ \\
\hline \multirow{5}{*}{ 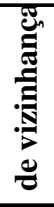 } & $\mathrm{ccv}$ & ----- & ---- & ----- & ----- & ----- & ----- & ----- & ----- \\
\hline & ccp & ----- & ----- & ----- & ----- & ----- & ----- & $0,0 \%$ & $(0,00006)$ \\
\hline & cld & ----- & $-\cdots$ & ----- & ----- & ----- & ----- & ----- & ----- \\
\hline & $\mathrm{ec}$ & ---- & ---- & $0,6 \%$ & 3.256 & ----- & ----- & ----- & ----- \\
\hline & pda & $0,0 \%$ & $(2.936)$ & $0,0 \%$ & $(4.502)$ & $0,0 \%$ & $(0,0073)$ & $0,0 \%$ & $(0,01191)$ \\
\hline & K-S empírico & \multicolumn{2}{|c|}{0,105} & \multicolumn{2}{|c|}{0,114} & \multicolumn{2}{|c|}{0,059} & \multicolumn{2}{|c|}{0,056} \\
\hline & K-S teórico & \multicolumn{2}{|c|}{0,046} & \multicolumn{2}{|c|}{0,046} & \multicolumn{2}{|c|}{0,046} & \multicolumn{2}{|c|}{0,046} \\
\hline
\end{tabular}

Os resultados indicam que a equação de regressão na função SEMI-LOG pelo método STEPWISE é a que apresenta estatísticas mais consistentes, se consideradas as premissas da análise de regressão. Por isso, foi escolhida para a análise das variáveis independentes e dos seus coeficientes. Observando as variáveis independentes, nota-se que quase todas as variáveis estruturais incluídas no modelo apresentam coeficientes de regressão com os sinais esperados (positivos) e significativos a 5\%. Nas variáveis de localização, há coeficientes com sinais contra-intuitivos: eram esperados sinais positivos para as variáveis Lagoa, Barra e Recreio, por serem bairros valorizados, e sinal negativo para a variável Tijuca. No caso das variáveis de vizinhança (variáveis criminais), as incluídas na equação (CCP e PDA) apresentam coeficientes negativos e significativos a 5\%, como esperado. 
Das variáveis estruturais, as que apresentaram menor índice de tolerância foram área $(0,28)$ e garagem $(0,38)$. Ambas foram incluídas no modelo de regressão pelo método STEPWISE, que nesse caso não filtrou todas as colinearidades. Apesar da colinearidade dessas variáveis, optou-se por mantê-las no modelo, por causa dos coeficientes estatisticamente significativos e com sinais conforme o esperado, mesmo sabendo do possível viés incorrido na análise.

Os resultados do modelo 1 estão em consonância com os obtidos por Cummings, Dipasquale e Kahn (2001), onde o aumento nos crimes, medido por assassinatos, resulta em um decréscimo nos preços dos imóveis. Esses autores utilizaram a forma SEMI-LOG em seu modelo de regressão, a mesma da equação de regressão escolhida para análise no modelo 1 . Os resultados também conferem com os encontrados por Clark e Cosgrove (1990), cujo modelo mostra que medidas de crimes são variáveis determinantes dos preços de imóveis urbanos. Assim, os resultados do modelo 1 sugerem que os números da violência criminal urbana são significativos e causam impacto negativo no valor dos imóveis. Ressalvada a fragilidade do modelo e dos dados e tomando cuidado para não extrapolar a amostra selecionada, o modelo indica que, para cada crime contra o patrimônio (CCP) no bairro do imóvel, há uma perda de $0,006 \%$ no seu valor, e também que para cada ocorrência de porte e disparo de armas (PDA), há uma perda de $1,2 \%$ no valor.

Vistos os principais resultados do modelo 1, podemos passar para os resultados obtidos com o modelo 2.

\section{Modelo 2}

A dispersão dos preços verificada nessa amostra de imóveis foi, naturalmente, menor do que no modelo 1. Levando em conta que ela cobre imóveis do mesmo bairro ou da mesma região, a segmentação em sub-regiões da Tijuca foi conseguida pela identificação do CEP (de @20260 a @ 20561). A tabela 6 resume alguns dos principais resultados conseguidos com as equações de regressão selecionadas. 
Tabela 6 - Resumo dos resultados modelo 2

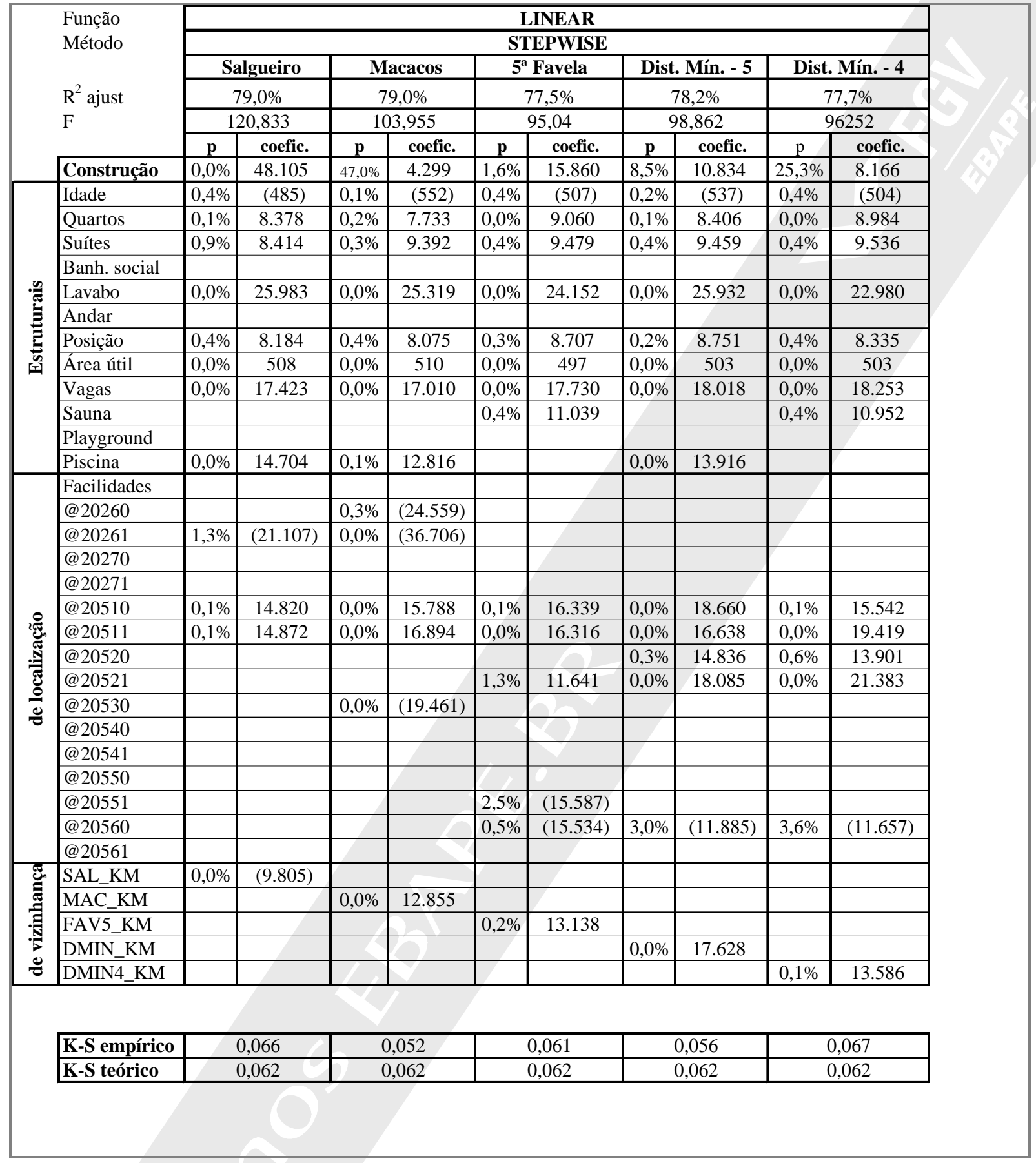

Analisando os coeficientes das regressões, observa-se que as variáveis estruturais apresentam sinais coerentes com os esperados e significativos a 5\%. Quanto às variáveis de localização (CEP), os resultados não são muito consistentes quanto à presença de homogeneidade entre as equações, exceto pelas variáveis @20510 e @ 20511, incluídas em todas as equações de regressão, com coeficientes significativos a 5\%. A variável FACILIDD (que representa proximidade do metrô) foi excluída de todas as regressões; indicando, provavelmente, má qualidade na escolha da variável ou na forma de mensuração.

Nas variáveis de vizinhança, observa-se consistência com as hipóteses do modelo 2. Todas as equações mostraram variáveis de vizinhança com sinais positivos e significativos a 5\%, exceto a variável SAL_KM, que apresentou sinal negativo para o coeficiente. Por outro lado, quando se examina a equação de regressão da 
variável DMIN_KM, esta é positiva e significativa. A variável DMIN_KM é a menor das distâncias medidas entre o imóvel e as cinco favelas (quatro de referência e a quinta, mais próxima, que não uma das quatro de referência). O mesmo acontece quando se toma o exemplo da equação da variável DMIN4_KM, que representa a menor entre as quatro distâncias (entre o imóvel e as quatro favelas de referência).

Na realidade, as variáveis DMIN_KM e DMIN4_KM são as que mais se aproximam da variável instrumental desejada para o modelo 2; ou seja, a menor distância, em linha reta, entre o imóvel e uma favela. Dessas variáveis, a DMIN_KM é a que representa a menor distância de todas as outras variáveis de vizinhança. Pelas razões anteriormente citadas e tanto pela qualidade das estatísticas gerais $\left(\mathrm{R}^{2}\right.$ ajustado, $F$ e KolmogorovSmirnov) quanto dos coeficientes das variáveis independentes dessa equação de regressão, optou-se pela distância mínima dentre as cinco medidas (DMIN_KM) para representar o modelo 2, com função LINEAR, calculada pelo método STEPWISE.

Devido às indicações de multicolinearidade entre as variáveis independentes, foi realizado um experimento para reduzir esse efeito: foram refeitas as regressões (LINEAR e STEPWISE) recorrendo-se não ao "preço" como variável independente, mas à razão "preço/ $\mathrm{m}^{2}$ ". Contudo, os resultados não justificaram a adoção dessa modelagem alternativa. Seria importante realizar análise mais específica sobre multicolinearidade em estudos posteriores.

Tendo o devido cuidado com a fragilidade dos resultados, há indicação no modelo 2 de que um imóvel na Tijuca perde cerca de $\mathrm{R} \$ 18.000,00$ no preço para cada quilômetro que se aproxima da favela mais próxima. Assim, reunindo os resultados dos modelos 1 e 2, a conclusão é de que um imóvel residencial na Tijuca sofre o efeito conjunto (talvez correlacionado) do número de crimes cometidos no bairro e da distância da área subnormal mais próxima. Utilizando os estimadores obtidos para os dois tipos de impacto, com as ressalvas decorrentes da imprecisão dos modelos, um imóvel na Grande Tijuca teria seu valor reduzido em cerca de 1,2\% por crime de posse e disparo de armas (PDA) cometido no bairro e em cerca de $\mathrm{R} \$ 18.000,00$ por quilômetro que se aproxime da favela mais próxima.

\section{Conclusão}

O objetivo deste estudo foi avaliar o impacto da violência criminal urbana no preço dos imóveis residenciais do bairro da Tijuca, município do Rio de Janeiro, utilizando dados do mercado imobiliário do período de junho de 2000 a janeiro de 2002. Trata-se de um estudo de natureza exploratória, cujos resultados devem ser considerados com muito cuidado.

A literatura relativa aos modelos de preços hedônicos mostra que não há uma indicação teórica consensual para a forma funcional das equações de regressão, nem procedimentos preferenciais claramente estabelecidos para o cálculo das regressões, ou clareza para a seleção das variáveis explicativas a serem incluídas nos modelos. Na verdade, a literatura sugere a experimentação através do método científico. Dessa perspectiva, os modelos concebidos nesta pesquisa buscaram adequar fontes e qualidade de dados (acessibilidade) aos preceitos do método de preços hedônicos. Logo, os resultados aqui obtidos precisam ser analisados com muita cautela.

O modelo 1 apresentou resultados que indicaram a influência negativa dos crimes de uma região no valor do imóvel ali situado. Esses resultados, compatíveis com o senso comum, encontram respaldo nos dados encontrados na literatura (CUMMINGS, DIPASQUALE e KAHN, 2001; CLARK e COSGROVE, 1990). Nesse caso, a função SEMI-LOG foi a forma funcional que melhor se adequou aos dados obtidos, em sintonia com o estudo de Cummings, Dipasquale e Kahn. Além disso, os resultados indicam que há uma segmentação entre os bairros da amostra e, conseqüentemente, diferentes funções de demanda para consumidores de imóveis de diferentes bairros, ainda que alguns coeficientes não apresentem os sinais esperados.

Os resultados do modelo 2 parecem confirmar outra impressão consagrada pelo senso comum; a de que quanto mais próximo da favela, menor o preço de um imóvel. Na literatura não foi encontrado um estudo que 
relacionasse a distância de uma área sub-normal ao impacto causado pela violência criminal urbana no preço dos imóveis residenciais. Por conseguinte, não há comparação entre os resultados e a literatura pesquisada.

De acordo com os modelos empregados, há um impacto negativo de cerca de $1,2 \%$ no preço do imóvel para cada crime que aconteça na área desse imóvel (resultado do modelo 1), mais um impacto negativo de cerca de R\$ 18.000,00 para cada quilômetro que o imóvel se aproxima da favela mais próxima na área da Tijuca (resultado do modelo 2). Conforme já foi observado, são números que devem ser considerados com a cautela. Como nos casos tratados na literatura pesquisada, não há como estabelecer, a priori, as variáveis instrumentais adequadas para medir o impacto da violência criminal urbana. Há indícios, tanto na literatura quanto neste estudo, de que as variáveis criminais por região (por bairros, como as empregadas no modelo 1, por setores censitários, por cidades etc.) apresentam resultados que se ajustam às hipóteses levantadas. Porém, mais importante que a variável teórica em si é a qualidade dos dados e das fontes.

Aparentemente, pode-se inferir que a proximidade de áreas de baixa renda e de elevados índices criminais influencia negativamente o valor dos imóveis; o que não fica claro é a relação de causa e efeito entre essas variáveis. A proximidade de uma área de baixa renda pode influenciar o valor dos imóveis por razões diferentes das explicitadas nos modelos desenvolvidos. Especificamente quanto à distância como variável instrumental, há necessidade de estabelecer, em bases empíricas mais sólidas, a relação entre a distância da área subnormal e a violência criminal urbana, ou, ainda, definir as variáveis endógenas presentes na variável "distância". O modelo 2 supõe que a variável distância tem apenas a componente da violência criminal, o que não é necessariamente verdade.

Portanto, é com extrema cautela que devemos considerar os resultados da pesquisa. Na verdade, os resultados específicos obtidos não são a maior contribuição desta pesquisa, devido à baixa qualidade de alguns dados e à falta de consenso na literatura sobre a modelagem quantitativa. As principais contribuições estão na indicação dos caminhos a serem seguidos, na descrição dos dados necessários e na orientação para elaboração de bases de dados adequadas ao desenvolvimento de novas pesquisas na área.

A questão da violência criminal urbana transcende o mercado imobiliário e, infelizmente, é parte do dia-a-dia das grandes cidades brasileiros, particularmente do Rio de Janeiro. Assim, estudos como este podem ser instrumentos para fornecer balanços de custos e benefícios úteis na formulação de políticas públicas. O custo social imposto pela baixa qualidade de vida, decorrente da falta de segurança, pode ser um elemento importante na formulação das estratégias de desenvolvimento econômico e planejamento urbano.

Como sugestões para futuras pesquisas nessa área, seria importante investigar de forma mais aprofundada as multicolinearidades entre as variáveis explicativas, identificando quais delas deveriam realmente ser incluídas nos modelos de regressão. Uma possível abordagem seria avaliar mais detalhadamente a variável dependente "preço/m2". O estudo do custo da violência criminal no valor dos imóveis também poderia incluir novos municípios, trazendo resultado no âmbito estadual ou mesmo nacional. Pela dificuldade de se conseguir dados nesse mercado, estudos metodológicos poderiam estabelecer regras ou políticas que sistematizassem a coleta e a disponibilização de informações atualizadas sobre negociações de imóveis, desenvolvendo sistemas que organizassem e racionalizassem os procedimentos para se conseguir informações no mercado imobiliário. 


\section{Referências bibliográficas}

BENSON, P. G.; M CCLAVE, J. T.; SINCICH, T. L. Statistics for business and economics. 8. ed. [S.I.]: MacGraw-Hill, 2002.

BOWEN, W. M.; MIKELBANK, B. A.; PRESTEGAARD, D. M. Theoretical and empirical considerations regarding space in hedonic housing price model applications. Growth and Change, v.32, n.4, p. 466-490, Fall 2001.

BRUEGGEMAN, W. B.; FISHER, J. D. Real estate finance and investments. 10. ed. Boston: Irwin MacGraw-Hill, 1997.

CLARK, D. E.; COSGROVE, J.C. Hedonic prices, identification, and the demand for public safety. Journal of Regional Science, v.30, n.1, p.105-121, 1990.

CUMMINGS, J. L.; DIPASQUALE, D.; KAHN, M. E. Measuring the consequences of promoting inner city homeownership. Working paper. City Research e Fletcher School - Tufts University. 2001. Disponível em: <www.cityresearch.com.br>. Acesso em: 31 jul. 2002.

DING, C.; SIMONS, R.; BAKU, E. The effect of residential investment on nearby property values: evidence from Cleveland, Ohio. Journal of Real Estate Research, v.19, n.1/2, p.23-48, 2000.

DIXON, J.; SCURA, L. F.; CARPENTER, R. A.; Sherman, P. B. Economic analysis of environmental impacts. 2. ed. London: Earthscan Publications, 1994.

FREEMAN III, A. M. The measurement of environmental and resource values: theory and methods. Washington, D.C.: Resources for the Future, 1993.

MACEDO, P. B. R. Hedonic price models with spatial effects: na application to the housing market of Belo Horizonte, Brazil. Revista Brasileira de Economia, Rio de Janeiro, v.52, n.1, p.63-81, jan./mar. 1998.

ROSEN, S. Hedonic prices and implicit markets: product differentiation in pure competition. Journal of Political Economy, v.82, n.1, p.3455, Jan./Feb. 1974.

TU, C. C.; EPPLI, M. J. Valuing new urbanism: the case of Kentlands. Real Estate Economics, v.27, n.3, p.425-451, 1999.

WILHELMSSON, M. The impact of traffic noise on the values of single-family houses. Journal of Environmental Planning and Management, v.43, n.6, p.799-815, 2000. 